\title{
Results on Inclusive Jets and Jet Properties
}

\author{
Mikko Voutilainen ${ }^{1,2,3}$ for the D0 Collaboration \\ 1- University of Nebraska in Lincoln - Dept. of Physics and Astronomy \\ Lincoln, NE 68588-0111 - USA \\ 2- Helsinki Institute of Physics - CMS Programme \\ P.O. Box 64, FIN-00014 University of Helsinki - Finland \\ 3- CEA-Saclay - Le service de Physique des Particules \\ F-91191 Gif-sur-Yvette Cedex - France
}

\begin{abstract}
We report on a measurement of the inclusive jet cross section in proton-antiproton collisions at a center-of-mass energy of $1.96 \mathrm{TeV}$ using data collected by the D0 experiment at the Fermilab Tevatron Collider corresponding to an integrated luminosity of $0.70 \mathrm{fb}^{-1}$. Detailed studies of correlations between systematic uncertainties in transverse momentum and rapidity are presented, and the cross section measurements are found to be in good agreement with next-to-leading order QCD calculations.
\end{abstract}

\section{Introduction}

The production of jets in hadron collisions is described by the theory of quantum chromodynamics (QCD). When the transverse jet momentum with respect to the hadron beam direction $\left(p_{T}\right)$ is large, the contributions from long-distance physics processes with low $p_{T}$ are small and the production rates of jets can be predicted by perturbative QCD (pQCD). The inclusive jet cross section in $p \bar{p}$ collisions at large $p_{T}$ is directly sensitive to the strong coupling constant $\left(\alpha_{s}\right)$ and the parton density functions (PDFs) of the proton. It provides one of the most direct probes of physics at small distance scales and potential deviations from the $\mathrm{pQCD}$ prediction at high $p_{T}$, not explained by the PDFs, may indicate new physics beyond the standard model.

\section{Jet energy scale}

Due to the steeply falling inclusive jet $p_{T}$ spectrum the measurement is very sensitive to the jet energy scale (JES). The jet energies are corrected to the particle level, i.e. they correspond to the energies of jets made out of stable particles after hadronization, using

$$
E_{\mathrm{ptcl}}=\frac{E_{\mathrm{cal}}-E_{\mathrm{off}}}{R \cdot S} \cdot k_{\mathrm{bias}}
$$

where $E_{\text {offset }}$ is the offset contribution, $R$ is the response of the particle jet, $S$ is the net showering due to detector effects and $k_{\text {bias }}$ is an explicit correction for bias in the databased methods. The jet energy scale corrects for "offset" energies measured using zerobias events, which correspond to calorimeter and electronics noise, pile-up effects (previous bunch-crossings) and multiple interactions (same bunch-crossing). The electromagnetic (EM) calorimeter is calibrated using the $Z$ boson peak in $Z \rightarrow e^{+} e^{-}$events. The energy scale of the EM calorimeter is transferred to photons in $\gamma+$ jet events, with the compensating photon purity and relative photon-electron energy scale shifts explicitly accounted for by using Monte Carlo (MC) with the electromagnetic shower simulation tuned to data.

DIS 2008 
The absolute JES in the central calorimeter is assigned using the transverse momentum balance between the jet and the photon in the $\gamma+$ jet events. The JES is different for jets in the dijet sample due to the physical differences of quark and gluon jets. This JES difference is computed using $\mathrm{MC}$ with the single pion response tuned to data using $\gamma+$ jet events. The same $\mathrm{MC}$ is used to extrapolate JES to high $p_{T}$. The detector pseudorapidity dependence $(\eta$ intercalibration) of JES is determined in a simultaneous fit using both dijet and $\gamma+$ jet events with one jet or photon central, while taking into account the sample differences. Detector effects cause some of the particle jet energy to be showered outside the cone, or outside energy to be showered inside the

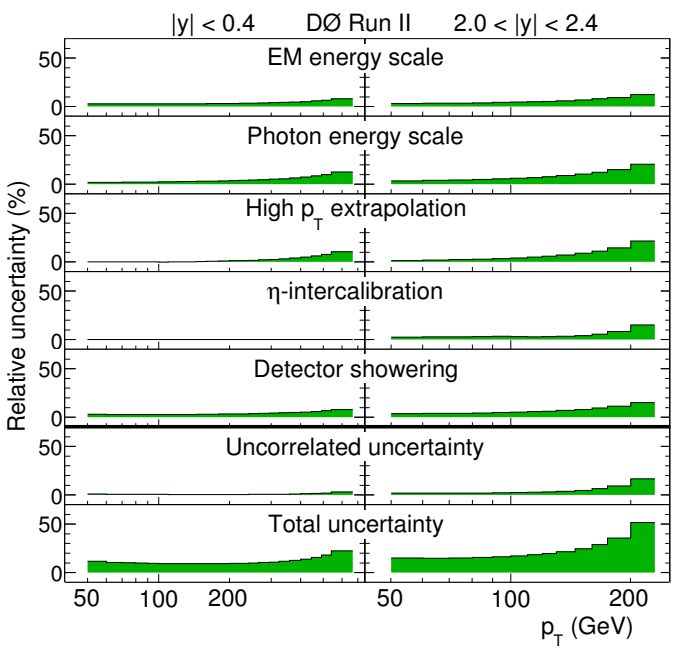
cone. The net effect is accounted for by measuring the energy density profile around the jet, subtracting from this the contribution to the showering due to physics effects from MC. Finally, $k_{\text {bias }}$ explicitly accounts for the small bias in the data-based methods due to zero suppression and topological effects. The final energy calibration has an uncertainty of $1.2-2.5 \%$, which is the most precise JES at hadron colliders to date, and the calibration uncertainties are strongly correlated across $p_{T}$ and jet rapidity $y$. The resulting cross section uncertainty correlations are provided as a set of 23 uncertainty sources, whose five leading contributions are from JES and are shown in Fig. 1. The uncorrelated systematic uncertainty is small compared to the total uncertainty.

\section{$3 \quad$ Jet $p_{T}$ resolution and unfolding}

The jet $p_{T}$ resolution is measured on a subsample of the same data set as is used for the cross section measurement using dijet asymmetry

$$
A=\frac{\left|p_{T, 1}-p_{T, 2}\right|}{p_{T, 1}+p_{T, 2}}
$$

which is corrected for soft radiation of additional jets below the jet reconstruction threshold and for particle level imbalance. The shape of the asymmetry distribution is taken from MC, which is useful to describe e.g. the non-Gaussian energy leakage of some high $p_{T}$ jets due to the finite depth of the calorimeter. The spectra in $p_{T}$ are fit, in an iterative procedure, with parameterized ansatz functions,

$$
f(N, \alpha, \beta, \gamma)=N\left(p_{T} / \mathrm{GeV}\right)^{-\alpha}\left(1-\frac{2 \cosh \left(y_{\min }\right) p_{T}}{\sqrt{s}}\right)^{\beta} \exp \left(-\gamma p_{T}\right),
$$

where $y_{\min }$ is the minimum absolute rapidity in the bin and the $\sqrt{s}$ is the center-of-mass energy, and folded with resolutions determined from data for $p_{T}$ and from MC for $y$. Ratios of the original to the folded ansatz functions are used to correct the data for folding of resolution effects.

DIS 2008 


\section{Results}

The cross section is measured using seven different triggers, which are matched together using relative trigger efficiencies. The cross section is corrected for jet identification and event selection efficiencies. The final results corrected for $p_{T}$ and $y$ resolutions are shown in Fig. 2. The agreement with pQCD is good over the wide $p_{T}$ and $y$ region explored. The leading uncertainty in the measurement is the JES, as shown in Fig. 2 for the $|y|<0.4$. The fully correlated $6.5 \%$ luminosity uncertainty also contributes at low $p_{T}$ in this region.
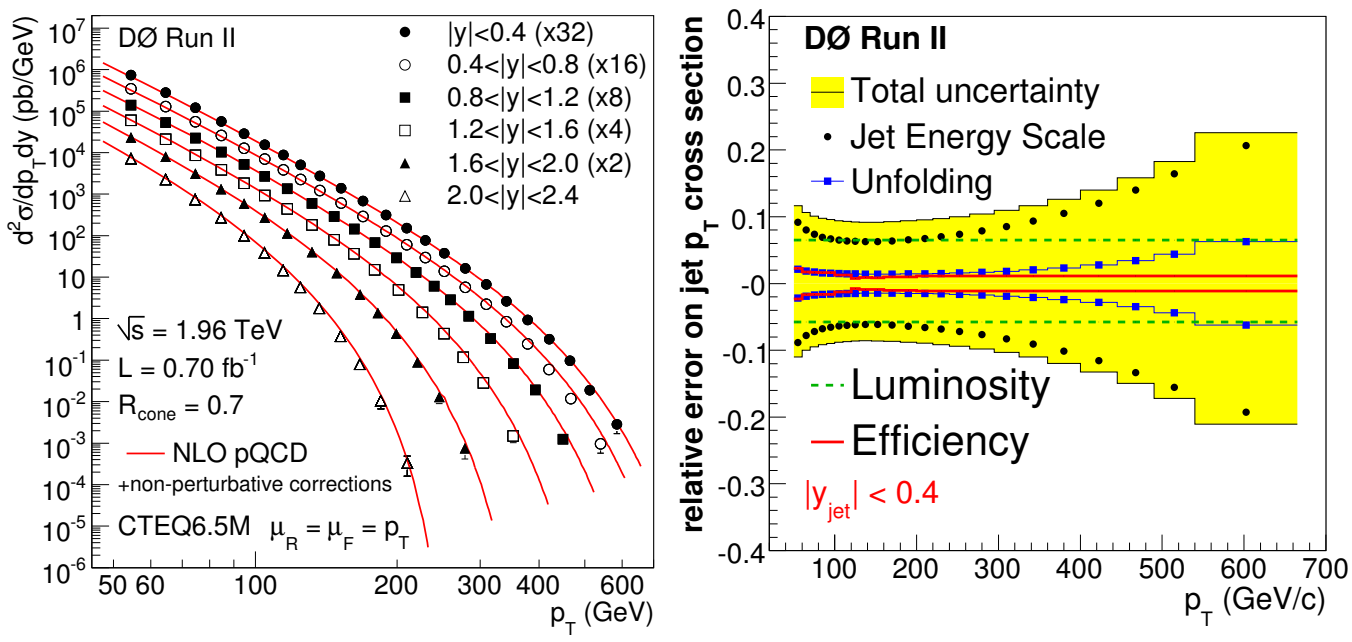

Figure 2: The inclusive jet cross section, measured in six regions of jet rapidity up to $|y|=2.4$ (left). Error bars show the total measurement uncertainty. The predictions from pQCD are corrected for hadronization effects and are overlaid on the data as lines. The total measurement uncertainty and its components in the central region $|y|<0.4$ (right).

The cross section measurement is compared to next-to-leading order (NLO) theory with non-perturbative corrections for hadronization and underlying event in Fig. 3 in six regions of jet rapidity. The NLO calculations were performed using NLOJet++ [2] and FastNLO [3]. The PDF uncertainty from CTEQ6.5M [4] is overlaid as short-dashed lines, showing that the measurement is precise enough to constrain the PDFs at high $p_{T}$. Most of the PDF uncertainty is coming from the uncertainty in the gluon PDFs at high momentum fraction $x$. The prediction using MRST2004 [5] PDFs is shown as a long-dashed line to compare the two PDF sets. The theory scale uncertainty, calculated as a half-difference between the predictions with $\mu_{R}=\mu_{F}=p_{T} / 2$ and $\mu_{R}=\mu_{F}=2 p_{T}$, is about $10 \%$ at all $p_{T}$ and $y$ as shown by the hatched band in Fig. 3 .

\section{Conclusions}

Results on the inclusive jet cross section measurements at D0 were shown with $0.70 \mathrm{fb}^{-1}$ of collected luminosity [6]. The measurement is sensitive to the parton distribution functions of the proton and allows one to reduce the gluon density uncertainty at high momentum

DIS 2008 


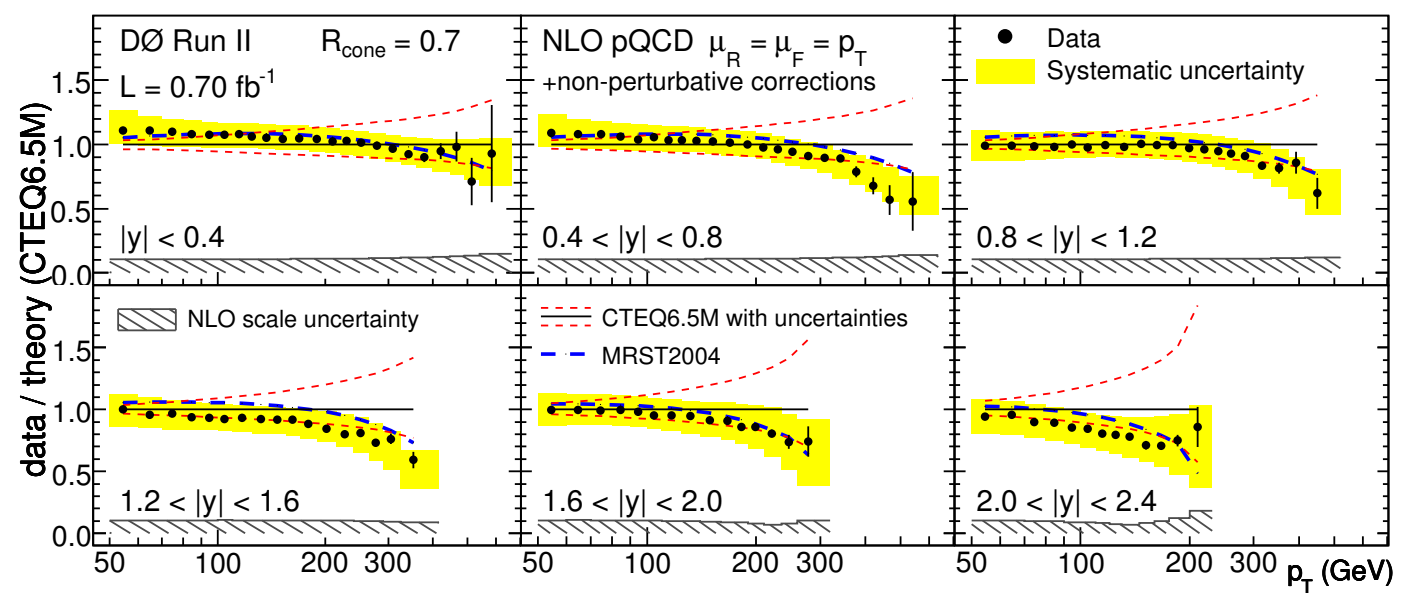

Figure 3: Ratio of data and the theory prediction using CTEQ6.5M PDF. The shaded band and bars show the measurement systematic and statistical uncertainties, respectively. The short dashes show the CTEQ6.5 PDF uncertainty and the long dashes the theory prediction using MRST2004 PDFs. The scale uncertainty at NLO is shown by the hatched band.

fraction. With improved jet energy scale determination, the D0 cross section measurement has the smallest experimental uncertainties to date at the Tevatron. The wide rapidity coverage and the uncertainty correlations further increase the impact of the data on the global parton distribution fits. The results are in good agreement with the next-to-leading order perturbative QCD calculations and favor the MRST2004 central prediction and the CTEQ6.5M prediction with reduced high- $x$ gluon.

\section{Acknowledgements}

I thank my colleagues at D0 and acknowledge the support from the Graduate School in Particle and Nuclear Physics, the Finnish Cultural Foundation and the Magnus Ehrnrooth Foundation.

\section{References}

[1] Slides: http: //indico. cern. ch/contributionDisplay . py? contribId=216\&sessionId=13\&conf Id=24657

[2] Z. Nagy, Phys. Rev. D68, 094002 (2003).

[3] T. Kluge, K. Rabbertz, M. Wobisch, arXiv:hep-ph/0609285 (2006).

[4] W. K. Tung et al., JHEP 0702, 053 (2007); J. Pumplin et al., JHEP 0207, 12 (2002); D. Stump et al., JHEP 0310, 046 (2003).

[5] A. D. Martin et al., Phys. Lett. B604, 61 (2004)

[6] V. M. Abazov et al., arXiv:0802.2400v1 [hep-ex] (2008). 